\title{
Analysis of Dual Sourcing Strategy with Quality Differentiated Suppliers
}

\author{
Shuang Zhao ${ }^{1}$ \\ ${ }^{1}$ School of Business Administration Guangdong university of Finance and Economics Guangzhou, China
}

\begin{abstract}
Co-opetition supply chain is now the normal mode of development among enterprises. Based on the co-opetition supply chain, this paper designs a model to study the procurement strategy between OEM and two suppliers with supply uncertainty. We find that the profit increase of OEM is less affected by the wholesale price of a single supplier when purchasing through a single channel suppress. The dual purchase strategy has a great advantage, which leads to price war and reduces wholesale price. However, it is interesting to find that when the technical level of competitive suppliers is constantly improving, the order quantity of competitive suppliers is not always rising, but presents a single peak mode. This shows that OEM can deal with the threat of profit by reducing the orders to competitive suppliers.
\end{abstract}

\section{Introduction}

Competition has spread from enterprises to supply chains. Competition-cooperation supply chains have become the most common mode during supply chain development in the $21^{\text {st }}$ century. For example, Samsung is the OEM of mobile phone processors and OLED screens of Apple. They are competitors in the downtown mobile phone market, but they are cooperators in the process of upstream part OEM manufacturing. Competition-cooperation supply chains are also very common in the household appliance industry. Pilot run of 5G began in 2019. Major household appliance enterprises speed up the progress of breaking down original barriers to promote interconnection and intercommunication of different brands of intelligent appliances. The suppliers of Xiaomi $\mathrm{AC}$ are TCL and Changhong.

There are also some risks in the competitioncooperation supply chain. On one hand, suppliers have many uncertain factors. For example, there are many uncontrollable factors in the agriculture, such as weather conditions, temperatures or natural disasters, so the crop yield is uncertain (yuan2020). In the semiconductor industry, the quality of components and parts may cannot meet the quality standard of OEM, or there is a problem of production technologies, so the actual output may be different from the initial output. On the other hand, unitary supplier will cause the strong pricing capacity, and purchase of components and parts from OEM will face risks (niu2019). As regards manufacturers, they also hope to access more other suppliers on the upstream to reduce the above risk. However, in the process of cooperating with several suppliers, it needs to be determined whether it is more competitive if OEM cooperate with more suppliers featured with different quality levels and low yield compared with cooperation with only a stable supplier and how OEM should distribute orders to such suppliers to maximize their own profits? To solve these problems, we design a model which simulates the upstream supply chain of enterprises in this study. This model contains an OEM and two suppliers whose productivity is uncertain (the uncertainty of their productivity is different). We respectively research three scenarios.

Our research mainly focuses on optimized production and purchase strategies of enterprises in the event of in stable supply. There are many literature about uncertain productivity. Lu Yaobin (2004) mentions in his literature that the supply uncertainty in the supply chain management context is mainly caused by the change of the production lead time, change of order quantity, uncertainty of the output time arising from production technologies of suppliers, etc. Gupta et al. (2014) researched a two-stage supply chain composed with two suppliers and two manufacturers. Wherein supplier I has a supply risk but a low wholesale price, supplier II is reliable but has a high wholesale price, and the manufacturer will purchase goods from both the supplier I and the supplier II to avoid risks.

In terms of purchase strategies, Niu et al. (2019) researched purchase problems of competition-cooperation supply chain dominated by OEM. The supply chain has competitive suppliers and non-competitive suppliers. Wherein, competitive suppliers provide products for OEM and manufacture final products which are similar to those of OEM and form competition with OEM in the downstream market. Jie et al. (2020) researched the case where supplied products had a quality problem by designing a model. In this model, there is an OEM and one supplier as well as a quantity-based pricing contract. Cao Yu (2021) analyzed impacts of the brand effect, simulation capacity of the competitive supplier and the raw material 
supply capacity of the non-competitive supplier on selection of the purchase mode and quality decision making. Both Yu(2021) and niu et al. (2019) carried out research on the competition-cooperation supply chain. The model of this paper is the extension version of the model of niu et al. (2019). This paper further takes into consideration whether the OEM will take different purchase strategies when the supply of both the competitive supplier and the non-competitive supplier in the competition-cooperation supply chain is uncertain.

\section{MODEL FORMULATION}

This paper studies the situation in a co-copetition supply chain,which is composed of an original equipment manufacturer (OEM), a competitive supplier (CS), and a non-competitive supplier (NS). However, there are differences in the quantity of parts supplied, and the quantity provided by competitive suppliers is higher than that provided by non competitive suppliers. We analyze the following three scenarios, as shown in Fig. 1 below.

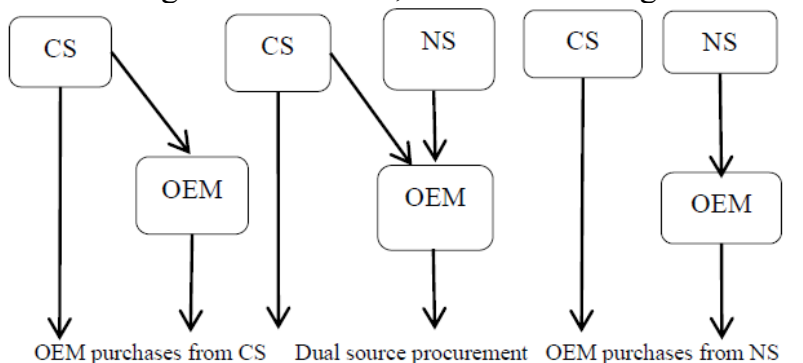

Figure .1 Model
In the first scenario, OEM has its own retail brand, and it will order raw materials from $\mathrm{CS}$, the competitive manufacturer decides the wholesale price $w_{c s}$ and OEM decides the quantity of products produced by its own brand is $q_{o}$. In this scenario, OEM only orders from CS, Competitive suppliers have uncertainty of supply quantity, the order quantity is $e_{1} q_{c s}, q_{c s}$ is the actual delivery quantity, $e_{1}$ is a random variable, and average value is $\mu$. We limit the range of random variable e to $[0,1], \sigma<(1 / 2)^{3}$. In the second scenario, because CS has strong pricing power, the price of raw materials is very high. Therefore, OEM will consider ordering some raw materials from other channels to reduce production costs. OEM will order some raw materials from NS which is not competitive in the market. Because the non-competitive suppliers also have the uncertainty of output, the order quantity is $e_{2} q_{n s}\left(e_{1}>e_{2}\right)$. OEM's output in the final market consists of two parts $q_{o}=e_{1} q_{c s}+e_{2} q_{n s}$. In the third scenario, with the increase of the technical level of non-competitive suppliers, OEM may abandon purchasing from competitive suppliers, all provided by non-competitive suppliers. Then NS decides the wholesale price $w_{n s}$ and the quantity of products $q_{o}=e_{2} q_{n s}$. In addition, consumers' demand for competitive suppliers' products is represented by a linear and downward sloping (inverse) demand function $\mathrm{p}_{c s}=a-b Q$, where $\mathrm{p}_{c s}$ is the retail price, $\mathrm{a}$ is the market potential, $\mathrm{b}$ is the quantity sensitivity, and $\mathrm{Q}$ is the total quantity available in the market. Without losing generality, we assume that the market potential a is large enough that the market demand is always positive. In order to capture the brand differences between products from OEM and competitive suppliers, we assume that the demand for OEMs' products is $\mathrm{p}_{c s}=a-b Q+m$, where $\mathrm{M}$ represents the brand differences $(\mathrm{m} \geq 0, \mathrm{a} \geq \mathrm{m})$ (niu2019).

\section{The Benchmark: Base Scenario}

This paper assumes that OEM and competitive supplier compete in Cournot market. The consumer demand for products is expressed by inverse demand function. Competitive suppliers determine the unit wholesale price, OEM places orders with competitive suppliers, and suppliers also determine the production quantity of their own brand products. Then the profit functions of CS and OEM are as follows:

$$
\begin{aligned}
& \Pi_{c s}=\left(a-b q_{b}-b e_{1} q_{c s}\right) q_{b}+w_{c s} e_{1} q_{c s} \\
& \Pi_{o}=\left(a+m-b q_{b}-b e_{1} q_{c s}\right) e_{1} q_{c s}-w_{c s} e_{1} q_{c s}
\end{aligned}
$$

LEMMA 1. In scenario 1, the equilibrium wholesale price, the equilibrium order quantity and the equilibrium expected profit can be obtained by calculation :

$$
\begin{aligned}
& w_{c s}=\frac{8 a \sigma_{1}{ }^{2}+5 a \mu_{1}{ }^{2}+8 m \sigma_{1}{ }^{2}+4 m \mu_{1}{ }^{2}}{16 \sigma_{1}{ }^{2}+10 \mu_{1}{ }^{2}} \\
& q_{b}=\frac{8 a \sigma_{1}{ }^{2}+5 a \mu_{1}{ }^{2}-2 m \mu_{1}{ }^{2}}{2 b\left(8 \sigma_{1}{ }^{2}+5 \mu_{1}{ }^{2}\right)} \\
& q_{c s}=\frac{2 m \mu_{1}}{b\left(8 \sigma_{1}{ }^{2}+5 \mu_{1}{ }^{2}\right)} \\
& E \Pi_{o}=\frac{4 m^{2} \mu_{1}{ }^{2}\left(\sigma_{1}{ }^{2}+\mu_{1}{ }^{2}\right)}{b\left(8 \sigma_{1}{ }^{2}+5 \mu_{1}{ }^{2}\right)^{2}} \\
& E \Pi_{c s}=\frac{8 a^{2}{\sigma_{1}}^{2}+5 a^{2} \mu_{1}{ }^{2}+4 m^{2} \mu_{1}{ }^{2}}{4 b\left(8 \sigma_{1}{ }^{2}+5 \mu_{1}{ }^{2}\right)}
\end{aligned}
$$

Because the competitive supplier has the uncertainty of production, we represent the technical level of competitive supplier by $x_{1}=\left(\mu_{1} / \sigma_{1}\right)^{2}$. We can see what strategy OEM will adopt, when the technical level of CS increases, by analyzing the function of $x 1$.

LEMMA 2. Obviously, the above equilibrium solution can be transformed into a function of $\mathrm{x}_{1}$, The following statistics are available .

$$
\frac{\partial w_{c s}}{x_{1}}>0 \frac{\partial q_{b}}{x_{1}}<0 \frac{\partial q_{c s}}{x_{1}}>0 \frac{\partial E \Pi_{o}}{x_{1}}>0 \frac{\partial E \Pi_{c s}}{x_{1}}>0
$$

PROPOSITION 1. As we expected, when the technology level of CS increases, the wholesale price will rise. However, the output of CS itself is decreasing, and the overall profit is increasing, which shows that the increase of orders of CS offsets the rise of wholesale price and the decrease of market production.

\section{The Dual Sourcing Scenario}

The second scenario describes that the OEM adopts a dual procurement strategy to allocate its component orders to competing suppliers and non-competing suppliers. Both supply chains have output uncertainty, and the uncertainty of competitive suppliers is less than that of noncompetitive suppliers, which are labeled as $\mathrm{e}_{1} \mathrm{q}_{\mathrm{cs}}$ and $\mathrm{e}_{2} \mathrm{qns}$ respectively, and OEM's total received component quantity is $\mathrm{q}_{\mathrm{o}}=\mathrm{e}_{1} \mathrm{q}_{\mathrm{cs}}+\mathrm{e}_{2} \mathrm{q}_{\mathrm{ns}}$, Then the profit function of the dual channel model is as follow: 


$$
\begin{aligned}
& \Pi_{c}=\left(a-b q_{b}-b\left(e_{1} q_{c s}+e_{2} q_{n s}\right) q_{b}+w e e_{c 1} q_{c S}\right. \\
& \Pi_{o}=\left(a+m-b q_{b}-b\left(e_{1} q_{S S}+e_{2} q_{n s}\right)\left(e_{1} q_{C S}+e_{2} q_{n s}\right)-w_{\mathrm{SS}} e_{1 S}-w_{n S} e_{n s}\right. \\
& \prod_{n s}=w_{n s} e_{2} q_{n s}
\end{aligned}
$$

In order to simplify the calculation results, we take $x_{1}=\left(\mu_{1} / \sigma_{1}\right)^{2}$ and $x_{2}=\left(\mu_{2} / \sigma_{2}\right)^{2}$ as the technical level of competitive suppliers and non-competitive suppliers, which is an indicator of the quality of their production process.It's straightforward to see that the decrease of $\delta$ or the increase of $\mu$ leads to a higher level of $x$. Some parameters are set according to the research of Niu et al. (2019). Market size $a=1$, quantity sensitivity $b=0.5$, brand difference $\mathrm{m}=0.6$, standard deviation $\sigma_{1}=0.18$, $\sigma_{2}=0.18$. Take these values into the calculation, and then carry out numerical analysis.

LEMMA 3. In dual sourcing scenario, All equilibrium solutions are formulas for $\mathrm{x}_{1}$ and $\mathrm{x}_{2}$, the equilibrium wholesale price, the equilibrium order quantity can be obtained by calculation :

$$
\begin{aligned}
& q_{b}=\frac{108 x_{1}^{2} x_{2}+456 x_{1}^{2}+108 x_{1} x_{2}^{2}+1188 x_{1} x_{2}+1568 x_{1}+456 x_{2}^{2}+1568 x_{2}+1280}{5\left(81 x_{1}^{2} x_{2}+120 x_{1}^{2}+81 x_{1} x_{2}^{2}+384 x_{1} x_{2}+352 x_{1}+144 x_{2}^{2}+384 x_{2}+256\right)} \\
& q_{c s}=\frac{\left(20 x_{1}^{1 / 2}\right)\left(144 x_{1}+320 x_{2}+186 x_{1} x_{2}+99 x_{2}^{2}+192\right)}{\left(9 \left(81 x_{1}^{2} x_{2}+120 x_{1}^{2}+81 x_{1} x_{2}^{2}+384 x_{1} x_{2}+352 x_{1}+144 x_{2}^{2}+384 x_{2}\right.\right.} \\
& \left(9\left(81 x_{1}^{2} x_{2}+120 x_{1}^{2}+81 x_{1} x_{2}^{2}+384 x_{1} x_{2}+352 x_{1}+144 x_{2}^{2}+384 x_{2}+256\right)\right) \\
& \left(100 x_{1}^{1 / 2}\right)\left(3 x_{1}+4\right)\left(\frac{37 x_{1}}{5}+\frac{65 x_{2}}{5}+\frac{88}{5}\right) \\
& q_{n s}=\frac{5}{\left(9\left(81 x_{1}^{2} x_{2}+120 x_{1}^{2}+81 x_{1} x_{2}^{2}+384 x_{1} x_{2}+352 x_{1}+144 x_{2}^{2}+384 x_{2}+256\right)\right)} \\
& \left(\frac{\left(6 x_{1}+6 x_{2}+8\right)\left(37 x_{1}\right)}{5}+\frac{66 x_{2}}{5}+\frac{88}{5}\right) \\
& w_{n s}=\frac{5}{\left.\left(81 x_{1}^{2} x_{2}+120 x_{1}^{2}+81 x_{1} x_{2}^{2}+384 x_{1} x_{2}+352 x_{1}+144 x_{2}^{2}+384 x_{2}+256\right)\right)}
\end{aligned}
$$

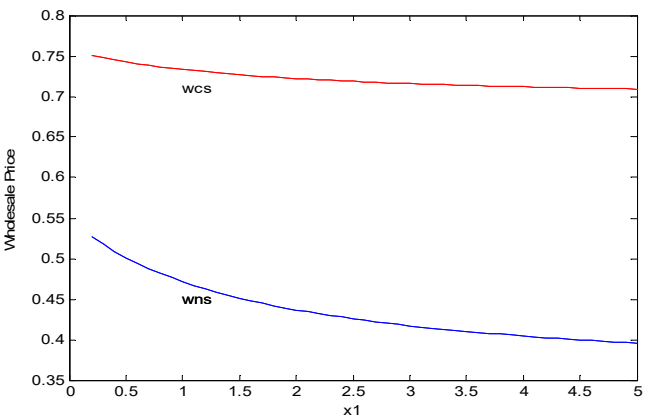

Figure .2 Wholesale price of NS and CS

PROPOSITION 2. 1. $\mathrm{W}_{\mathrm{cs}}$ and $\mathrm{w}_{\mathrm{ns}}$ are decreasing in $\mathrm{x}_{1 . ;}$ Qcs is unimodal in $\mathrm{x} 1$ for all given feasible $\delta$.

As shown in Fig. 2, when we assume that the technical level of $\mathrm{NS}_{2} \quad\left(\mathrm{x}_{1}>\mathrm{x}_{2}\right)$ remains stable, we give a value $(\mathrm{x} 2=0.1)$, and the technical level of CS $\mathrm{x}_{1}$ is gradually increasing, which will pose a threat to NS. Therefore, we can see that the response of NS is to significantly reduce the wholesale price of $\mathrm{w}_{\mathrm{ns}}$, and at the same time induce a corresponding small reduction of $\mathrm{CS}\left(\mathrm{w}_{\mathrm{cs}}>\mathrm{w}_{\mathrm{ns}}\right)$, Under the dual channels, the price war was induced.

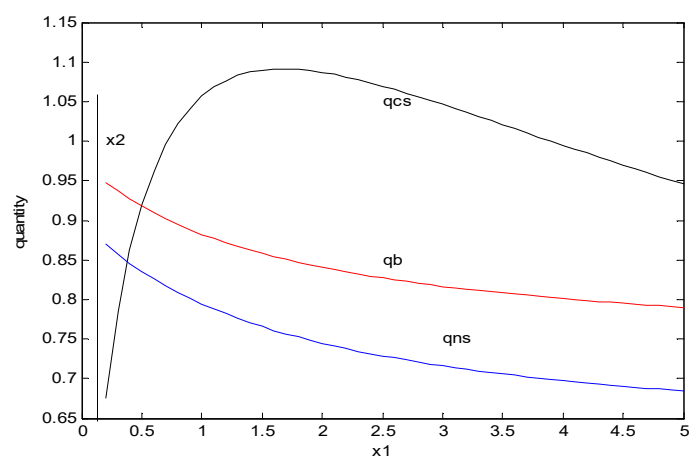

Figure .3Sales volume of CS and orders of NS and CS

The information we can get from Fig. 3 and Fig. 4 is that when the uncertainty of CS is decreasing, that is, the technical level is improving, the order quantity of CS is increasing, and the order quantity of NS is decreasing, which is in line with the actual law. The increase of the quality of the supplier will bring him more order quantity. However, interestingly, we can see that the order quantity of $\mathrm{q}_{\mathrm{cs}}$ does not increase monotonously, but in a single peak mode. When the order quantity of $\mathrm{q}_{\mathrm{cs}}$ reaches a peak, the order quantity begins to decrease. To understand this finding, we further investigate how the expected profit and the wholesale prices change inthe technology level $\mathrm{x}_{1}$. Interestingly, We have the following result of proposition 3.

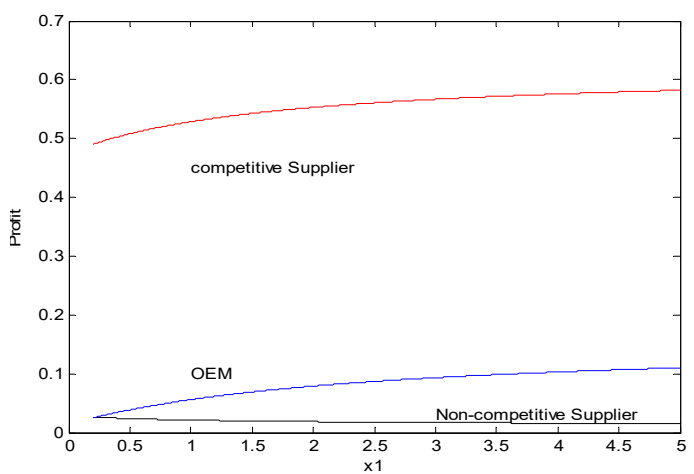

Figture .4 Profits of CS and NS

PROPOSITION 3.

When the OEM adopts the dual sourcing strategy, the non-competitive supplier's expected profit decreases in $\mathrm{x}$; The competitive supplier 's expected profit is more than the OEM 's expected profit.

From the change of CS's order quantity, we find that when the technical level of suppliers has been increasing, OEM does not always increase its order quantity, but decreases after reaching the peak. This can be explained by the change of the profit function of the two. With the increase of $x 1$, dual channel purchasing will increase the profits of OEM and CS, but we find that the profits of CS are much higher than that of OEM. Because OEM and CS are competitors, if the order volume of competitors is increased, the total profit of competitors will be increased constantly.

\section{The Termination Scenario}

In this scenario, the OEM sources solely from the noncompetitive supplier. non-competitive supplier determines the component wholesale price wns, The output of NS is uncertain, so its final order quantity is $\mathrm{e}_{2}$ qns. CS does not provide OEM with raw material orders at this time, and its main profit source is the sales volume 
of downstream product market. Then the profit functions ofCS , NS and OEM are as follows:

$$
\begin{aligned}
& \Pi_{c s}=\left(a-b q_{b}-b\left(e_{2} q_{n s}\right) q_{b}\right. \\
& \Pi_{o}=\left(a+m-b q_{b}-b e_{2} q_{n s}\right) e_{2} q_{n s}-w_{n s} e_{2} q_{n s} \\
& \Pi_{n s}=w_{n s} e_{2} q_{n s}
\end{aligned}
$$

LEMMA 4.In the termination scenario, the equilibrium wholesale price, the equilibrium order quantity and the equilibrium expected profit can be obtained by calculation :

$$
\begin{aligned}
& w_{n s}=\frac{a+2 m}{4} \\
& q_{b}=\frac{8 a \sigma_{2}^{2}+(5 a-2 m) \mu_{2}{ }^{2}}{16 b \sigma 2^{2}+12 b \mu_{2}{ }^{2}} \\
& q_{n s}=\frac{(a+2 m) \mu_{2}}{8 b \sigma 2^{2}+6 b \mu_{2}{ }^{2}} \\
& E \Pi_{o}=\frac{(a+2 m)^{2} \mu_{2}^{2}\left(\sigma_{2}{ }^{2}+5 \mu_{2}{ }^{2}\right)}{4 b\left(4 \sigma_{2}{ }^{2}+3 \mu_{2}{ }^{2}\right)^{2}} \\
& E \Pi_{c s}=\frac{\left(8 a \sigma_{2}{ }^{2}+(5 a-2 m) \mu_{2}\right)^{2}}{16 b\left(4 \sigma_{1}{ }^{2}+3 \mu_{1}{ }^{2}\right)^{2}} \\
& E \Pi_{n s}=\frac{(a+2 m)^{2} \mu^{2}}{8 b\left(4 \sigma^{2}+3 \mu^{2}\right)^{2}}
\end{aligned}
$$

This part of the model is similar to the model of Niu et al. (2019), and the results are directly explained by the results of his article. we find that the OEM 's production quantity is increasing while the competitive supplier's production is decreasing in the brand difference $m$. The OEM 's profit as well as the non-competitive supplier's profit both increase in $\mathrm{m}$. Without the component-selling business, there is no trade off and the competitive supplier's profit will decrease in m.Regarding the impact of the non-competitive supplier's production technology level.

\section{CONCLUSION REMARKS AND FUTURE RESEARCH}

This paper establishes a competitive supply chain composed of OEM, CS and NS, and studies the purchasing decision-making problem of OEM when both suppliers have supply uncertainty. From the perspective of OEM, the optimal procurement strategy is not necessarily to adopt dual source procurement at any time. From scenario 2 , we can see that when CS technology is increasing, OEM's profit is also increasing. At this time, dual source purchasing is beneficial to OEM. Because OEM can reduce the cost of procurement and enhance the competitiveness in the product market. Under dual source procurement, the two suppliers will reduce the wholesale price in order to compete for orders of raw materials. In the process of continuous improvement of NS raw material supply capacity, the price reduction trend of raw materials of the two suppliers is more obvious, and the growth trend of OEM profit is also more significant. However, with the continuous rise of competitive suppliers' technical level, when the total profit of the suppliers is gradually widening the gap with OEM, OEM will face the threat of CS at this time. The total profits of $\mathrm{CS}$ are increasing, increasing the company's capital and increasing the competitiveness of downstream products. This paper is based on the article of dual channel procurement strategy of competitive supply chain by neu et al. (2019), and an extended study is conducted. The original study is an uncertain supplier. For the OEM procurement mode, this paper considers that both suppliers may have uncertainty of production, which may be close to the real life scenario. However, there are still many deficiencies in this paper. Future research can consider more factors that affect the uncertainty of suppliers, as well as solutions, and the impact of brand differences between competing partners under the basic model of competitive supply chain. These are the directions worthy of in-depth exploration.

\section{REFERENCES}

1. Xiao yong Yuan, Gong bing Bi, Ya lei Fei , Lin dong Liu. Supply chain with random yield and financing[J]. Omega, 2020.

2. Baozhuang Niu ,Jiawei Li, Jie Zhang, Hsing Kenneth, ChengYinliang (Ricky) Tan . Strategic Analysis of Dual Sourcing and Dual Channel with an Unreliable Alternative Supplier[J]. Production and Operations Management, 2019, 28(3) : 570-587.

3. Lu Yaobin, Yang Guangming, Yang Mincai. Sources, performance and Countermeasures of uncertainty in supply chain management [J]. Progress and Countermeasures of science and technology, 2004 (04): 87-89.

4. Tang, S.H. Gurnani, D. Gupta. 2014. Managing disruptions in decentralized supply chains with endogenous supply process reliability. Prod. Oper. Manag. 23: 1198-1211.

5. Jie Gao, Huirong Fana, Bin Caoc, Nengmin Wang. Quality incentive contracts considering asymmetric product manufacture ability information: Piece rate vs. Tournament $[\mathrm{J}]$. Computers \& Industrial Engineering, 2020, 144

6. Baozhuang Niu, Zihao Mu. Sustainable efforts, procurement outsourcing, and channel co-opetition in emerging markets $[\mathrm{J}]$. Transportation Research Part E,2020,138.

7. Cao Yu, Wei Panpan, Li Qingsong. Research on procurement and quality decision making in competitive supply chain [J / OL]. China ManagementScience:113[2020328]https://doi.org/10 .16381/j.cnki.issn1003-207x.2019.2113.

8. Jiguang Chen and $\mathrm{He} \mathrm{Xu}$ and Pin Zhou. Delegation vs. direct sourcing revisited: contract types under correlated supply risks and asymmetric cost information[J]. International Journal of Production Research, 2019, 58(22) : 7005-7022.

9. Wu, X., F. Zhang. 2014. Home or overseas? An analysis of sourcing

strategies under competition. Management Sci. 60: 1 $223-1240$. 